\title{
Total Exchange Routing on Hierarchical Dual-Nets
}

\author{
Yamin $\mathrm{Li}^{1}$ and Wanming $\mathrm{Chu}^{2}$ \\ ${ }^{1}$ Hosei University, Tokyo 184-8584 Japan \\ 2 University of Aizu, Aizu-Wakamatsu 965-8580 Japan
}

\begin{abstract}
The hierarchical dual-net (HDN) is a newly proposed interconnection network for massive parallel computers. The HDN is constructed based on a symmetric product graph (base network). A $k$-level hierarchical dual-net, $\operatorname{HDN}(B, k, S)$, contains $n_{k}=\left(2 n_{0}\right)^{2^{k}} /\left(2 \prod_{i=1}^{k} s_{i}\right)$ nodes, where $S=\left\{G_{1}^{\prime}, G_{2}^{\prime}, \ldots, G_{k}^{\prime}\right\}, G_{i}^{\prime}$ is a super-node and $s_{i}=\left|G_{i}^{\prime}\right|$ is the number of nodes in the super-node at the level $i$ for $1 \leq i \leq k$, and $n_{0}$ is the number of nodes in the base network $B$. The $S$ is used mainly for adjusting the scale of the system. The node degree of $\operatorname{HDN}(B, k, S)$ is $d_{0}+k$, where $d_{0}$ is the node degree of the base network. The HDN is node and edge symmetric and can contain huge number of nodes with small node-degree and short diameter. The total exchange is one of the most dense communication patterns and is at the heart of numerous applications and programming models in parallel computing. In this paper, we show that the total exchange routing can be done on HDN efficiently.
\end{abstract}

Keywords: interconnection network, total exchange routing.

\section{Introduction}

Recently, because of the advances in computer and networking technologies, supercomputers containing hundreds of thousands of nodes have been built [10]. It was predicted that the parallel systems of the next decade will contain 10 to 100 millions of nodes [2]. The interconnection network plays an important role for achieving high-performance in such ultra-scale parallel systems. The performance of an ultra-scale parallel computers depends largely on the time complexities of communication schemes, and in turn depends on the diameter of the network. An interconnection network consists of switches with multiple communication ports and cables connecting ports by following certain topologies. For an ultrascale parallel computer, the traditional interconnection networks may no longer satisfy the requirements for the high-performance computations or efficient communications. For such an ultra-scale parallel computer, the node degree and the diameter will be the critical measures for the effectiveness of the interconnection networks. The node degree is limited by the hardware technologies and the diameter affects all kinds of communication schemes directly. The number of communication ports (node degree) in the network-on-chip (NoC) is typically 4 to 8 in current implementations. The off-chip interconnect switches can have 
tens of ports, but the cost becomes expensive as the number of ports increases. Other important measures for the effectiveness of the interconnection networks include symmetricity, scalability, and efficient routing algorithms.

The following two categories of interconnection networks have attracted a great research attention and been used in many supercomputers' implementations. One is the hypercube-like family that has the advantage of short diameters for high-performance computing and efficient communications. The other is the $2 \mathrm{D} / 3 \mathrm{D}$ mesh or torus family that has the advantage of small and fixed node degrees and easy implementations [1]. Traditionally, most supercomputers including those built by CRAY, IBM, SGI, and Intel use 3D tori or hypercubes. However, the node degree of the hypercube increases logarithmically as the number of nodes in the systems increases; the diameter of the $2 \mathrm{D} / 3 \mathrm{D}$ torus becomes large in an ultra-scale parallel system. To solve these problems, the hierarchical (cluster-based) architectures are proposed in literature [36. The supercomputer Roadrunner built by IBM adopts a new approach for the interconnection network [4. It is a cluster-based architecture: the connection among clusters is fully connected, and the fat-tree is used for the connection inside a cluster.

In this paper, we first present a flexible interconnection network, called $\mathrm{Hi}$ erarchical Dual-Net (HDN) [8]. The HDN is symmetric and can connect a large number of nodes with a small node degree, meanwhile keeping the diameter short. The HDN was motivated by recursive dual-net (RDN) [7. The RDN can be viewed as a special case of HDN. The RDN has merits of low node degree and short diameter. The problem of the RDN is that it grows too fast in size, and there is no mechanism to control the rate of its growth. Different from the RDN, the scale of the HDN can be controlled by setting a set of suitable parameters while generating an expanded network through dual-construction. The HDN also adapts the cluster-based architecture. Compared to the Roadrunner, the HDN is symmetric, uses small number of links, and meanwhile keeps the diameter short. The HDN structure is also better than other popular existing networks such as hypercube and $2 \mathrm{D} / 3 \mathrm{D}$ torus with respect to the degree and diameter. We investigate the topological properties of the HDN and show some examples of HDNs with simple base networks of small size. Then we compare them to other networks such as three-dimensional torus used in IBM Blue Gene/L [1, and hypercube. The total exchange, or all-to-all personalized communication, is one of the most dense communication patterns and is at the heart of numerous applications and programming models in parallel computing. In this paper, we present an efficient total exchange routing algorithm on a hierarchical dual-net. The time complexity $T_{k}(m)$ of the algorithm for an $\operatorname{HDN}(B, k, S)$ is $T_{k}(m)=\left(2^{k+1}-2\right)\left(t_{s}+t_{w} m n_{k} / 2\right)+2^{k} T_{0}\left(m n_{k} / 2\right)$, where $n_{k}$ is the total number of nodes, $t_{s}$ is startup latency, $m$ is the message length in words, and $t_{w}$ is the per-word transfer time.

The rest of this paper is organized as follows. Section 2 introduces the hierarchical dual-net in details. Section 3 describes the routing algorithm. Section 4 gives the total exchange routing algorithm on a hierarchical dual-net. Section 5 concludes the paper. 


\section{The Hierarchical Dual-Net}

We begin with a brief introduction to the recursive dual-net (RDN). The RDN is constructed recursively by a dual-construction. The dual-construction is a way to expand a given symmetric graph $G$ of size $n$ to a new symmetric graph $G^{*}$ of size $2 n^{2}$. It generates $2 n$ copies of $G$ as subgraphs (denoted as clusters) of $G^{*}$. Half of them, $n$ clusters, are of class 0 and the others are of class 1 . The connection method is described below.

If $G$ is symmetric then the expanded graph $G^{*}$ is unique and symmetric. Therefore, the dual-construction can be applied recursively from a symmetric network (the base network). $\operatorname{RDN}(m, k)$ denotes an RDN generated from a base network of size $m$ by applying dual-construction $k$ times. The problem about an RDN is that its growth rate is super-exponential $\left((2 m)^{2^{k}}\right)$. There is very little space for selection of the size of an RDN. For example, let the base network be a 3 -cube, then the sizes of $\operatorname{RDN}(8, k)$ will be $2^{7}, 2^{15}$, and $2^{31}$ for $k=1,2$, and 3 , respectively. In HDN, we provide a mechanism to control the growth rate through its expansion from a base network. This new interconnection network has a very flexible way for adjusting its size.

The hierarchical dual-net, $\operatorname{HDN}(B, k, S)$, contains three sets of parameters: $B$ is a symmetric product graph, we call it base network; $k$ is an integer that indicates the level of the HDN (the number of dual-constructions applied); and $S=\left\{G_{1}^{\prime}, G_{2}^{\prime}, \ldots, G_{k}^{\prime}\right\}$, where $G_{i}^{\prime}$ is a sub-graph of $\operatorname{HDN}(B, k-1, S)$ and $s_{i}=\left|G_{i}^{\prime}\right|$ is the number of nodes in a super-node at the level $i$ for $1 \leq i \leq k$. All these terminologies will be defined in the following paragraphs.

Given $r$ graphs $G_{i}=\left(V_{i}, E_{i}\right), 1 \leq i \leq r$, their product graph $G=G_{1} \times G_{2} \times$ $\ldots \times G_{r}$ is defined as the graph $G=(V, E)$, where $V=\left\{\left(v_{1}, v_{2}, \ldots, v_{r}\right) \mid v_{i} \in V_{i}\right.$, $1 \leq i \leq r\}$ and $E=\left\{\left[\left(u_{1}, u_{2}, \ldots, u_{r}\right),\left(v_{1}, v_{2}, \ldots, v_{r}\right)\right] \mid\right.$ for some $j,\left(u_{j}, v_{j}\right) \in E_{j}$ and for $\left.i \neq j, u_{i}=v_{i}\right\}$.

In other words, the nodes of the product graph $G$ are labeled with $r$-tuples, where the $i$ th element of the $r$-tuples is chosen from the node set of the $i$ th component graph. The edges of the product graph connect pairs of nodes whose labels are identical in all but the $j$ th element, and the two nodes corresponding to the $j$ th elements in the $j$ th component graph are connected by an edge.

Meshes/tori or hypercubes are typical examples of product graphs. Given a product graph $G=G_{1} \times G_{2} \times \ldots \times G_{r}$, we define a quotient graph $Q$ as $Q=G / G^{\prime}$ where $G^{\prime}$ is a sub-product graph of $G$ such that $G=G^{\prime} \times Q$. A node in a product graph $G=G_{1} \times \ldots \times G_{i} \times \ldots \times G_{r}$ can be represented by $\left(a_{1}, \ldots, a_{i}, \ldots, a_{r}\right)$ with $0 \leq a_{i} \leq\left|G_{i}\right|-1$. We define a sub-graph $G^{\prime}$ as $G^{\prime}=G_{1}^{\prime \prime} \times \ldots \times G_{j}^{\prime \prime} \times \ldots \times G_{q}^{\prime \prime}$ with $G_{j}^{\prime \prime}=G_{i}$ for $1 \leq j \leq q \leq r$ and $1 \leq i \leq r, G_{j}^{\prime \prime} \neq G_{k}^{\prime \prime}$ if $j \neq k$ for $1 \leq j, k \leq q$. Then a node in the sub-graph $G^{\prime}$ can be represented by $\left(b_{1}, \ldots, b_{i}, \ldots, b_{q}\right)$ with $0 \leq b_{i} \leq\left|G_{i}^{\prime \prime}\right|-1$. We can consider a quotient graph $Q$ as a reduced graph of $G$ with $G^{\prime}$ being mapped into a single node (a super-node).

A graph $G$ is symmetric (node-symmetric) if all its nodes looks alike. A product graph is symmetric if all its component graphs are symmetric. We use the symmetric product graph as the base network for generating a hierarchical dual-net 
through dual-constructions. We denote the base network as $B=B_{1} \times B_{2} \times \ldots \times B_{r}$ where all the $B_{i}, 1 \leq i \leq r$, are symmetric. We define a super-node of $B$, denoted as $S N$ as a sub-product graph of $B$. That is, $S N=B_{i_{1}} \times B_{i_{2}} \times \ldots \times B_{i_{q}}$, where $i_{j}, 1 \leq j \leq q$, are distinct and $q \leq r$.

Let $\left|B_{i}\right|=b_{i}$ be the number of nodes in $B_{i}$ for $1 \leq i \leq r$. The $\operatorname{HDN}(B, 0, S)=$ $B$ is the base network. For $i>0$, the $\operatorname{HDN}(B, i, S)$ is generated from $\operatorname{HDN}(B, i-$ $1, S)$ by a construction to be explained below. Note that $S=\left\{G_{1}^{\prime}, G_{2}^{\prime}, \ldots, G_{k}^{\prime}\right\}$, where $G_{i}^{\prime}$ is a sub-graph of $\operatorname{HDN}(B, k-1, S)$ and $s_{i}=\left|G_{i}^{\prime}\right|$ is the number of nodes in a super-node at the level $i$ for $1 \leq i \leq k$. First, we define a super-node of level $i$, denoted as $S N^{i}$, to be a sub-product graph $G_{i}^{\prime}$ of size $s_{i}$ in $B$. Then, we define graph $Q^{i}$ as the quotient graph $\operatorname{HDN}(B, i-1, S) / S N^{i}$. Suppose that there are $N_{i-1}$ nodes in the $\operatorname{HDN}(B, i-1, S)$, then the number of nodes $n_{i}$ in $Q^{i}$ is $N_{i-1} / s_{i}$. The $s_{i}$ can be 1 or $\prod_{j=1}^{q}\left|B_{i_{j}}\right|$, where $1 \leq i_{j} \leq r$ and $q \leq r$. That is, $s_{i}$ can be a product of any number of integers in $\left\{b_{1}, b_{2} \ldots, b_{r}\right\}$. For example, if $r=3, b_{1}=2, b_{2}=3$, and $b_{3}=5$, the possible $s_{i}$ can be $1,2,3,5,2 \times 3,2 \times 5$, $3 \times 5$, or $2 \times 3 \times 5$.

The construction of $\operatorname{HDN}(B, i, S), 1 \leq i \leq k$, can be defined by a two-step process: First, we perform a dual-construction on the quotient graph $Q^{i-1}=$ $\operatorname{HDN}(B, i-1, S) / S N^{i}(\operatorname{HDN}(B, 0, S)=B)$. Let the graph generated by the dualconstruction be $Q^{i}$, and the subgraph of two nodes that is connected by a crossedge of level $i$ be $K_{2}$. Second, to get the $\operatorname{HDN}(B, i, S)$, we replace every $K_{2}$ in $Q^{i}$ by a product graph $K_{2} \times S N$. We call $\operatorname{HDN}(B, i-1, S)$ cluster of $\operatorname{HDN}(B, i, S)$.

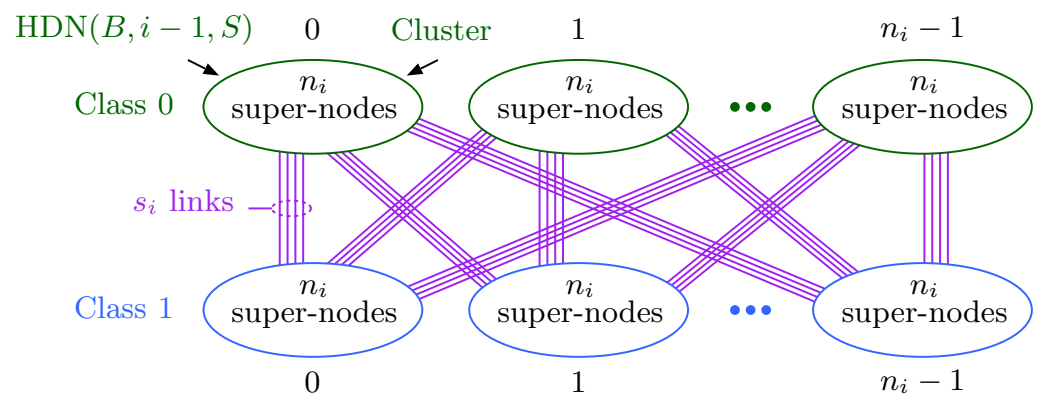

Fig. 1. Build an $\operatorname{HDN}(B, i, S)$ from $\operatorname{HDN}(B, i-1, S)$

Referring to Figure 11 an $\operatorname{HDN}(B, i, S)$ consists of $2 n_{i}$ clusters which are divided into two classes: class 0 and class 1 with each class containing $n_{i}$ clusters. That is, the number of clusters in each class is equal to the number of supernodes in a cluster. At level $i$, each super-node in a cluster has $s_{i}$ new links to a super-node in a distinct cluster of the other class. Because there are $s_{i}$ nodes in a super-node, one node contributes a new link. The dual-construction of an RDN is a special case of the construction of an HDN with $s_{i}=1$ for $1 \leq i \leq k$.

The indexes of the nodes in $\operatorname{HDN}(B, k, S)$ can be defined as follows. Let $S N_{i d}^{k}$ be a super-node_id in a cluster of $\operatorname{HDN}(B, k, S)$ and $N_{i d}^{k}$ be a node_id in a supernode, then a node in the $\operatorname{HDN}(B, k, S)$ can be represented by $\left(C^{k}, U_{i d}^{k}, S N_{i d}^{k}, N_{i d}^{k}\right)$ 
where $C^{k}$ is the class_id (0 or 1$)$ and $U_{i d}^{k}$ is the cluster_id. A cross-edge at level $k$ connects node $\left(C^{k}, U_{i d}^{k}, S N_{i d}^{k}, N_{i d}^{k}\right)$ and node $\left(\overline{C^{k}}, S N_{i d}^{k}, U_{i d}^{k}, N_{i d}^{k}\right)$.

If we use a $2 \times 3 \times 5$ torus as the base network, Table 1 lists the number of nodes in $\operatorname{HDN}(B, 1, S)$ and $\operatorname{HDN}(B, 2, S)$ under the different configurations of $S$. The node degrees are 7 and 8 for $\operatorname{HDN}(B, 1, S)$ and $\operatorname{HDN}(B, 2, S)$, respectively, because the node degree of $B$ is 6 . From the table, we can see that the HDN covers the nodes range from several hundreds to several millions.

Table 1. Number of nodes in $\operatorname{HDN}(B, k, S)$ where $B$ is a $2 \times 3 \times 5$ torus

\begin{tabular}{c|rrrrrrrr}
\hline$k=1$ & $s_{1}=1$ & $s_{1}=2$ & $s_{1}=3$ & $s_{1}=5$ & $s_{1}=6$ & $s_{1}=10$ & $s_{1}=15$ & $s_{1}=30$ \\
\hline & 1,800 & 900 & 600 & 360 & 300 & 180 & 120 & 60 \\
\hline$k=2$ & $s_{2}=1$ & $s_{2}=2$ & $s_{2}=3$ & $s_{2}=5$ & $s_{2}=6$ & $s_{2}=10$ & $s_{2}=15$ & $s_{2}=30$ \\
\hline$s_{1}=1$ & $6,480,000$ & $3,240,000$ & $2,160,000$ & $1,296,000$ & $1,080,000$ & 648,000 & 432,000 & 216,000 \\
\hline$s_{1}=2$ & $1,620,000$ & 810,000 & 540,000 & 324,000 & 270,000 & 162,000 & 108,000 & 54,000 \\
\hline$s_{1}=3$ & 720,000 & 360,000 & 240,000 & 144,000 & 120,000 & 72,000 & 48,000 & 24,000 \\
\hline$s_{1}=5$ & 259,200 & 129,600 & 86,400 & 51,840 & 43,200 & 25,920 & 17,280 & 8,640 \\
\hline$s_{1}=6$ & 180,000 & 90,000 & 60,000 & 36,000 & 30,000 & 18,000 & 12,000 & 6,000 \\
\hline$s_{1}=10$ & 64,800 & 32,400 & 21,600 & 12,960 & 10,800 & 6,480 & 4,320 & 2,160 \\
\hline$s_{1}=15$ & 28,800 & 14,400 & 9,600 & 5,760 & 4,800 & 2,880 & 1,920 & 960 \\
\hline$s_{1}=30$ & 7,200 & 3,600 & 2,400 & 1,440 & 1,200 & 720 & 480 & 240 \\
\hline
\end{tabular}

Suppose that the node degree of the base network $B$ is $d_{0}$, the node degree of the $\operatorname{HDN}(B, k, S)$ is $d_{0}+k$. Let $N_{i-1}$ be the number of nodes in the $\operatorname{HDN}(B, i-$ $1, S)$. There are $N_{i}=2\left(N_{i-1} / s_{i}\right) N_{i-1}=2 N_{i-1}^{2} / s_{i}$ nodes in the $\operatorname{HDN}(B, i, S)$ for $1 \leq i \leq k$, where $N_{i-1} / s_{i}$ is the number of clusters in each class. That is, the number of nodes in the $\operatorname{HDN}(B, k, S)$ is $\left(2 n_{0}\right)^{2^{k}} /\left(2 \prod_{i=1}^{k} s_{i}\right)$, where $n_{0}$ is the number of nodes in the base network.

Let the diameter of the $\operatorname{HDN}(B, i-1, S)$ be $D_{i-1}$ and the diameter of the super-node $(\mathrm{SN})$ be $D\left(S N_{i}\right)$. Then, if we map a super-node into a single node, the diameter of the quotient graph $Q^{i-1}$ is $D\left(Q^{i-1}\right)=D_{i-1}-D\left(S N^{i}\right)$.

To route a node $u$ in a cluster of class 0 (or 1 ) to a node $v$ in a different cluster of the same class, we can route $u$ along with a direct link of level $i$ to a node $u^{\prime}$ in a cluster of class 1 (or 0 ). This takes one step. Then, we route $u^{\prime}$ inside the cluster to a node $w^{\prime}$ that can reach a node $w$ in the same cluster of node $v$ along with direct link of level $i$. The longest distance between nodes $u^{\prime}$ and $w^{\prime}$ is $D\left(Q^{i-1}\right)$.

Similarly, we can route node $w^{\prime}$ to a node $w$ (by one step) and then to a node $v^{\prime}$ which is in the same super-node of $v$ (by $D\left(Q^{i-1}\right)$ steps). Finally, we route $v^{\prime}$ to node $v$, this takes $D\left(S N^{i}\right)$ steps. Therefore, we have the following recurrence:

$$
D_{i}=2\left(1+D\left(Q^{i-1}\right)\right)+D\left(S N^{i}\right)=2 D_{i-1}-D\left(S N^{i}\right)+2
$$

Solving the above recurrence, we get the diameter $D_{k}$ of $\operatorname{HDN}(B, k, S)$ as below:

$$
D_{k}=2^{k} D(B)-\sum_{j=0}^{k-1} 2^{j} D\left(S N^{k-j}\right)+2^{k+1}-2
$$


where $D(B)$ and $D\left(S N^{i}\right), 1 \leq i \leq k$, are the diameters of the base network and the super-nodes, respectively. The results of the analysis in this section are summarized in the following theorem.

Theorem 1. Assume that the base network B is a symmetric, product graph and $S N^{i}, 1 \leq i \leq k$, are sub-product graphs of $B$ with $\left|S N^{i}\right|=s_{i}$. Let the number of nodes, the node-degree, and the diameter of $B$ be $n_{0}, d_{0}$, and $D_{0}$, respectively. Let the diameters of $S N^{i}, 1 \leq i \leq k$, be $D\left(S N^{i}\right)$. Let $S=\left\{G_{1}^{\prime}, G_{2}^{\prime}, \ldots, G_{k}^{\prime}\right\}$, where $G_{i}^{\prime}$ is a sub-graph of $\operatorname{HDN}(B, k-1, S)$ and $s_{i}=\left|G_{i}^{\prime}\right|$ is the number of nodes in a super-node at the level $i$ for $1 \leq i \leq k$. Then, the number of nodes of $\operatorname{HDN}(B, k, S)$ is $\left(2 n_{0}\right)^{2^{k}} /\left(2 \prod_{i=1}^{k} s_{i}\right)$, the node-degree $i s d_{0}+k$, and the diameter is $D_{k}=2^{k} D(B)-\sum_{j=0}^{k-1} 2^{j} D\left(S N^{k-j}\right)+2^{k+1}-2$, where $N$ is the number of nodes in $H D N(B, k, S)$.

Table 2 lists the topological properties of the torus, $n$-cube, CCC [9], DualCube [6], RDN, and HDN. The CCC (cube-connected cycles) is obtained by replacing a node in an $n$-cube with an $n$-node cycle. The Dual-Cube is a special case of RDN with $k=1$ and a base network of an $n$-cube.

Table 2. Comparison of topological properties

\begin{tabular}{c||c|c|c}
\hline Network & \# of nodes & Degree & Diameter \\
\hline \hline 3D Torus & $x * y * z$ & 6 & $(x+y+z) / 2$ \\
\hline$n$-cube & $2^{n}$ & $n$ & $n$ \\
\hline CCC $(n)$ & $n * 2^{n}$ & 3 & $2 n+\lfloor n / 2\rfloor-2$ \\
\hline Dual-Cube $(n)$ & $2^{2 n-1}$ & $n$ & $2 n$ \\
\hline $\operatorname{RDN}(m, k)$ & $(2 m)^{2^{k}} / 2$ & $d_{0}+k$ & $2^{k} * D_{0}+2^{k+1}-2$ \\
\hline $\operatorname{HDN}(B, k, S)$ & $(2|B|)^{2^{k}} /\left(2 \prod_{i \underline{\underline{s}_{1}}}^{k}\right)$ & $d_{0}+k$ & $2^{k}\left(D(B)-\sum_{j=0}^{k-1} 2^{j}\left(D\left(S N^{k-j}\right)\right)+2^{k+1}-2\right.$ \\
\hline
\end{tabular}

In [7, we introduced the $C R$ (cost ratio) for measuring the combined effects of the hardware cost (node degree) and the software efficiency (diameter) of an interconnection network. Instead of $C R$, this paper uses a more general measure, namely weighted cost ratio $C R_{w}(G)$, for the evaluation. The $C R_{w}(G)$ is defined as below. Let $|(G)|, d(G)$, and $D(G)$ be the number of nodes, the node degree, and the diameter of $G$, respectively. We define $C R_{w}(G)$ as

$$
C R_{w}(G)=\frac{w_{1} d(G)+w_{2} D(G)}{\log _{2}|(G)|}
$$

where $w_{1}$ and $w_{2}$ are weights for node degree and diameter, respectively. We have $w_{1}+w_{2}=100 \%$.

The weighted cost ratio $C R_{w}$ of an $n$-cube is always 1 regardless of its size and weights. The $C R_{w}$ for some $\operatorname{HDN}(B, k, S)$ is shown in Table 3 where $B$ is a $2 \times 3 \times 5$ torus and we assume $w_{1}=w_{2}=50 \%$. For simplicity, we use the number of nodes in super-nodes to represent $S$, instead of sub-graphs. From the table, we can see that the HDNs are more effective than hypercubes and tori measured by the weighted cost ratio although as the $s_{i}$ increases, the $C R_{w}$ becomes larger. 
Table 3. $C R_{w}$ with $w_{1}=w_{2}=50 \%$ for some $\operatorname{HDN}(B, k, S)$

\begin{tabular}{c||c|c|c|c}
\hline Network & $n$ & $d$ & $D$ & $C R$ \\
\hline \hline 10-cube & 1,024 & 10 & 10 & 1.00 \\
\hline 3D-Tori $(10)$ & 1,000 & 6 & 15 & 1.05 \\
\hline HDN $(B, 1,(1))$ & 1,800 & 7 & 10 & 0.79 \\
\hline HDN $(B, 1,(2))$ & 900 & 7 & 9 & 0.82 \\
\hline HDN $(B, 1,(3))$ & 600 & 7 & 9 & 0.87 \\
\hline \hline 19-cube & 524,288 & 19 & 19 & 1.00 \\
\hline 3D-Tori $(80)$ & 512,000 & 6 & 120 & 3.32 \\
\hline HDN $(B, 2,(2,2))$ & 810,000 & 8 & 19 & 0.69 \\
\hline HDN $(B, 2,(2,5))$ & 324,000 & 8 & 18 & 0.71 \\
\hline HDN $(B, 2,(5,2))$ & 129,600 & 8 & 17 & 0.74 \\
\hline
\end{tabular}

The minimum $C R_{w}$ shown in the list is 0.69 . Unfortunately, we do not know the theoretical or experimental optimal value of $C R_{w}$ up to the date we wrote this paper and it can be an open question for the future.

\section{Routing on HDN}

Given two nodes $u$ and $v$ in $\operatorname{HDN}(B, k, S)$, we first present a simple routing algorithm that finds a shortest path from $u$ to $v$. In Section 2, we defined the product and quotient graphs. Now, we define the difference graph as follows. Let $S N_{1}$ and $S N_{2}$ are two super-nodes in base network $B$, the difference graph $S N_{1}-S N_{2}$ is the sub-product graph of $B$ such that $B_{i}, 1 \leq i \leq r$, is in $S N_{1}-S N_{2}$ if and only if $B_{i} \subset S N_{1}$ and $B_{i} \not \subset S N_{2}$. For example, if $B=C_{2} \times C_{3} \times C_{5}$, $S N_{1}=C_{2} \times C_{3}$, and $S N_{2}=C_{3} \times C_{5}$ then $S N_{1}-S N_{2}=C_{2}$.

We also need a re-indexing process of nodes in the cluster, which is an $\operatorname{HDN}(B, i-1, S)$, for routing via cross-edges of level $i$ since the indexes of nodes in $\operatorname{HDN}(B, i-1, S)$ is based on $S N^{i-1}$ and the cross-edge of level $i$ is defined based on $S N^{i}$. The index of a node in $\operatorname{HDN}(B, i-1, S)$ contains four parts $\left(C^{i-1}, U_{i d}^{i-1}, S N_{i d}^{i-1}, N_{i d}^{i-1}\right)$ as explained in the previous section.

At the construction of the $i$ th level, $\operatorname{HDN}(B, i-1, S)$ becomes a cluster containing only two parts, $S N_{i d}^{i}$ and $N_{i d}^{i}$, of the node index in $\operatorname{HDN}(B, i, S)$. The other two parts, $C^{i}$ and $U_{i d}^{i}$, are generated from the construction at the $i$ th level. The re-indexing process that generates a 1-to-1 mapping between $\left(C^{i-1}, U_{i d}^{i-1}, S N_{i d}^{i-1}, N_{i d}^{i-1}\right)$ and $\left(S N_{i d}^{i}, N_{i d}^{i}\right)$ on an $\operatorname{HDN}(B, i-1, S)$ is necessary for the proposed routing algorithm.

Since the number of super-nodes $S N^{i}$ in $\operatorname{HDN}(B, i-1, S)$ equals to $N_{i-1} / s_{i}$, the range of $S N_{i d}^{i}$ is $2\left|U^{i-1} /\left(S N^{i}-S N^{i-1}\right)\right| \times\left|\left(S N^{i-1}-S N^{i}\right)\right|$. If $s_{i-1}=s_{i}$ then the re-indexing is simple: 1-1 mapping between $S N_{i d}^{i}$ and the 3-tuple $\left(C_{i d}^{i-1}, U_{i d}^{i-1}, S N_{i d}^{i-1}\right)$. However, when $s_{i-1} \neq s_{i}$, the re-indexing is a little complicated and is explained below.

Let the $q$-tuple, $\left(b_{i_{1}}, \ldots, b_{i_{q}}\right)$ be the index of a node in a super-node $S N$, where $b_{i_{1}} \times \ldots \times b_{i_{q}}=|S N|$. Then the re-indexing from $\left(C^{i-1}, U_{i d}^{i-1}, S N_{i d}^{i-1}, N_{i d}^{i-1}\right)$ 
to $\left(S N_{i d}^{i}, N_{i d}^{i}\right)$ moves the indexes of those $B_{j} \subset S N^{i}-S N^{i-1}$ into $N_{i d}^{i}$ and the indexes of those $B_{j} \subset S N^{i-1}-S N^{i}$ into $S N_{i d}^{i}$. For example, let $B=$ $C_{2} \times C_{3} \times C_{5}, s_{1}=\left|C_{2}\right| \times\left|C_{3}\right|=6$, and $s_{2}=\left|C_{3}\right| \times\left|C_{5}\right|=15$, then, the nodes in $\operatorname{HDN}(B, 1, S)$ can be represented by $\left(C^{1}, U_{i d}^{1}, S N_{i d}^{1}, N_{i d}^{1}\right)$, where $C^{1}=0$ or 1 , $0 \leq U_{i d}^{1}<5,0 \leq S N_{i d}^{1}<5$, and $0 \leq N_{i d}^{1}<6$. For the indexes of the nodes in $\operatorname{HDN}(B, 2, S)$, we perform re-indexing of nodes in $\operatorname{HDN}(B, 1, S)$, which is a cluster of $\operatorname{HDN}(B, 2, S)$, to get $\left(S N_{i d}^{2}, N_{i d}^{2}\right)$, where $0 \leq S N_{i d}^{2}<2 \times 5 \times 2=20$, and $0 \leq N_{i d}^{2}<3 \times 5=15$, obtained by swapping $\left|B_{1}\right|$ and $\left|B_{3}\right|$. That is, $\left|S N^{2}\right|=\left|C^{1}\right| \times\left|U^{1}\right| \times\left|B_{1}\right|=2 \times 5 \times 2=20$, and $\left|N^{2}\right|=\left|B_{2}\right| \times\left|B_{3}\right|=15$.

Table 4 shows four examples of re-indexing in detail for a cluster in the $\operatorname{HDN}(B, 2, S)$ with $B=C_{2} \times C_{3} \times C_{5}, s_{1}=2 \times 3=6$, and $s_{2}=3 \times 5=15$. In the $\operatorname{HDN}(B, 1, S)$, the node representation $\left(C^{1}, U_{i d}^{1}, S N_{i d}^{1}, N_{i d}^{1}\right)$ can be converted to a serial number $i$ by $i=C^{1} \times\left(|B| / s_{1}\right)^{2} \times s_{1}+U_{i d}^{1} \times\left(|B| / s_{1}\right)^{1} \times s_{1}+S N_{i d}^{1} \times s_{1}+N_{i d}^{1}=$ $C^{1} \times 150+U_{i d}^{1} \times 30+S N_{i d}^{1} \times 6+N_{i d}^{1}$. Similarly, the $\left(S N_{i d}^{2}, N_{i d}^{2}\right)$ can be converted to a number $S N_{i d}^{2} \times s_{2}+N_{i d}^{2}=S N_{i d}^{2} \times 15+N_{i d}^{2}$.

Table 4. Re-indexing examples

\begin{tabular}{c|c||c|c}
\hline \multicolumn{2}{c||}{ Index in $\operatorname{HDN}(B, 1, S)$} & \multicolumn{2}{c}{ Index in $\operatorname{HDN}(B, 2, S)$} \\
\hline$\left(C^{1}, U_{i d}^{1}, S N_{i d}^{1}, N_{i d}^{1}\right)$ & Serial number & $\left(S N_{i d}^{2}, N_{i d}^{2}\right)$ & Serial number \\
\hline \hline$(0,0,0,0)$ & $0 \times 150+0 \times 30+0 \times 6+0=0$ & $(0,0)$ & $0 \times 15+0=0$ \\
\hline$(1,4,2,3)$ & $1 \times 150+4 \times 30+2 \times 6+3=285$ & $(19,0)$ & $19 \times 15+0=285$ \\
\hline$(0,0,2,2)$ & $0 \times 150+0 \times 30+2 \times 6+2=14$ & $(0,14)$ & $0 \times 15+14=14$ \\
\hline$(1,4,4,5)$ & $1 \times 150+4 \times 30+4 \times 6+5=299$ & $(19,14)$ & $19 \times 15+14=299$ \\
\hline
\end{tabular}

Assume that the point-to-point routing algorithm in the base network is available. The proposed algorithm for routing node $u$ to node $v$ in $\operatorname{HDN}(B, k, S)$ works as follows. We first perform re-indexing of $u$ and $v$ if $k>1$. Then, there are three cases: the two nodes are in the same cluster (Case 1), in the distinct clusters of the same class (Case 2), and in the distinct clusters of distinct classes (Case 3 ). Case 1 is trivial. Case 3 can be reduced to Case 2 by routing $u$ via a cross-edge of level $k$. Therefore, we explain only the Case 2: The two nodes are in the distinct clusters with the same class. We first identify the super-nodes, denoted as $S N_{u^{\prime}}^{k}$ and $S N_{v^{\prime}}^{k}$, in the two $Q^{k-1}$ s containing $u$ and $v$, respectively, such that $S N_{u^{\prime}}^{k}$ and $S N_{v^{\prime}}^{k}$ are connected by a unique cross-edge of level $k$ in $Q^{k}$ from the dual-construction. Then, we route node $u$ to node $u^{\prime}$, and node $v$ to node $v^{\prime}$ inside the clusters of level $k$, respectively. Notice that, $u^{\prime}$ and $v^{\prime}$ are not unique although $S N_{u^{\prime}}^{k}$ and $S N_{v^{\prime}}^{k}$ are unique. The algorithm finds the $u^{\prime}$ and $v^{\prime}$ that leave $u_{3}^{k}$ and $v_{3}^{k}$ unchanged if possible. And then, the routing from $u$ to $v$ is done by routing $u^{\prime}$ to $u^{\prime \prime} \in S N_{v^{\prime}}^{k}$ via a cross-edge of level $k$ in $\operatorname{HDN}(B, k, S)$ and routing from $u^{\prime \prime}$ to $v^{\prime}$ inside $S N_{v^{\prime}}^{k}$. The algorithm is formally presented as Algorithm 1. The correctness of the algorithm and its time complexity are given in Theorem 2 ,

Theorem 2. Assume that the routing algorithms in the base network $B$ is available. In $\operatorname{HDN}(B, k, S)$ for $k>0$, routing between any two nodes can be done 


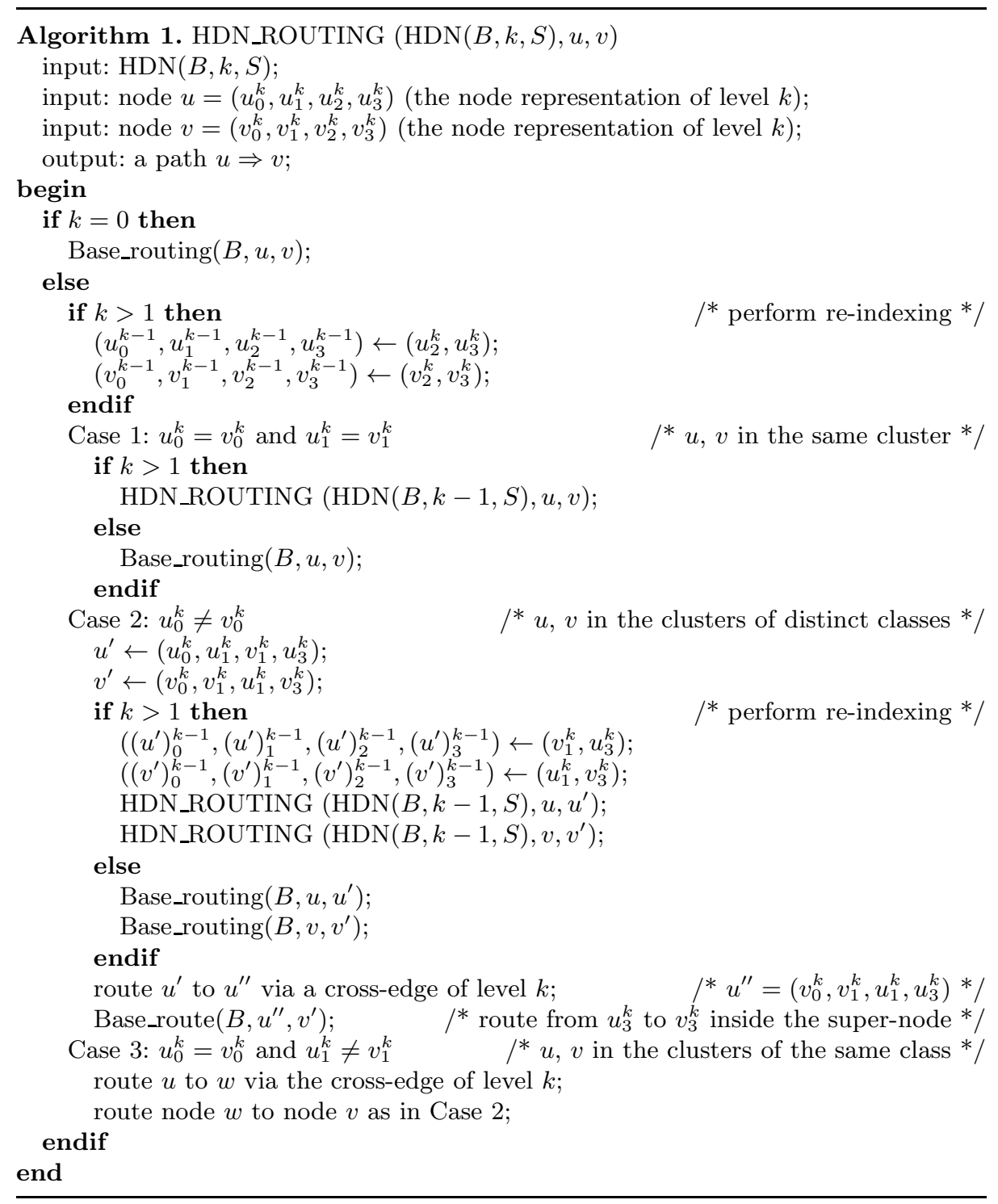

in at most $2^{k} R(B)-\sum_{j=0}^{k-1} 2^{j} R\left(S N^{k-j}\right)+2^{k+1}-2$ steps, where $R(B)$ and $R\left(S N^{i}\right), 1 \leq i \leq k$, are the time complexities of the routing in $B$ and $S N^{i}$, respectively.

Proof: We show the correctness of Algorithm 1 by induction on $k$. Assume that the algorithm is correct for $k-1 \geq 0$. From the algorithm, it is clear that we need to consider only Case 2 . In Case 2 , nodes $u^{\prime}$ and $u$ are in the same cluster by the definition of $u^{\prime}$. They can be connected by the induction hypothesis. Similarly, nodes $v^{\prime}$ and $v$ can be connected. The node $u^{\prime \prime}$ that is connected 
to $u^{\prime}$ by a cross-edge of level $k$ and node $v^{\prime}$ are in the same super-node as can be seen from their IDs. Therefore, they can be connected by Base_routing algorithm. Next, we derive the time complexity $R_{k}$ of the algorithm. In Case 2 , there are two recursive calls to connect $u$ to $u^{\prime}$ and $v$ to $v^{\prime}$, respectively. Since the nodeIDs of $u$ and $u^{\prime}$ are the same (so are $v$ and $v^{\prime}$ ), a recursive call takes only $R_{k-1}-R\left(S N^{k}\right)$ time. Since the SupernodeIDs of $u^{\prime \prime}$ and $v^{\prime}$ are the same, the last call to Base_route to connect $u^{\prime \prime}$ to $v^{\prime}$ takes only $R\left(S N^{k}\right)$ time. In Case 3 , there is an additional routing step via a cross-edge. Therefore, the time complexity $R_{k}$ of HDN_Routing $(\operatorname{HDN}(B, k, S), u, v)$ satisfies the recurrence $R_{k}=2\left(R_{k-1}-R\left(S N^{k}\right)\right)+R\left(S N^{k}\right)+2$ for $k>0$. Solving this recurrence, we have $R_{k}=2^{k} R(B)-\sum_{j=0}^{k-1} 2^{j} R\left(S N^{k-j}\right)+2^{k+1}-2$ where $R(B)$ and $R\left(S N^{i}\right), 1 \leq i \leq k$, are the time complexities of the routing in $B$ and $S N^{i}$, respectively.

\section{Total Exchange Routing on HDN}

Design of efficient routing algorithms for collective communications is the key issue in parallel computers or networks. Collective communications are required in load balancing, event synchronization, and data exchange. Based on the number of sending and receiving processors, these communications can be classified into one-to-many, one-to-all, many-to-many and all-to-all. The nature of the messages to be sent can be classified as personalized or non-personalized (multicast or broadcast). The all-to-all personalized communication (total exchange) is at the heart of numerical applications.

An important metric used to evaluate efficiency of communication is transmission latency, or communication time. The communication time depends on many factors such as contentions, switching techniques, network topologies etc. Therefore, we first define the communication model used in this paper.

We assume that the communication links are bidirectional, that is, two directly-connected processors can send messages to each other simultaneously. We also assume the processor-bounded model (one-port model) in which each node can access the network through a single input port and a single output port at a time. The port model of a network system refers to the number of internal channels at each node. In order to reduce the complexity of communication hardware, many systems support one-port communication architecture. We also assume the linear cost model in which the transfer time for a message is linearly proportional to the length of the message.

There are many switching methods. In this paper, we assume the packet switching model [5]. In this model, each packet is maintained as an entity that is passed from node to node as it moves through the network. The long message can be partitioned and transmitted as fixed-length word $w$. The first few bytes of a packet contains routing and control information and are referred as packet header. A packet is completely buffered at each intermediate node before it is forwarded to the next node (for this reason, the model is also called store-andforward switching). In this paper, we allow packages that are headed for the same destination to be combined into a single message. The time to pack and 
unpack messages is included in the startup latency. The packet switching model is suitable for collective communication in MPP since it is safer than other switching models such as virtual cut-through switching. With packet switching model, the communication time for a message of length $m$ (number of fixedlength words) to be sent to a node of distance $d$ is $d\left(t_{s}+m t_{w}\right)$, where $t_{s}$ is startup latency, the time required for the system to handle the message at the sending node, $t_{w}$ is the per-word transfer time $\left(1 / t_{w}\right.$ is the bandwidth of the communication links). Through this paper, we will use the formula above for estimating the communication times of the proposed algorithms.

In total exchange, each node sends a distinct message of size $m$ to every other node. The total number of messages is $p^{2}$ (a node also has a message for itself). Referring to Figures 2 and 3, the algorithm for total exchange in HDN can be

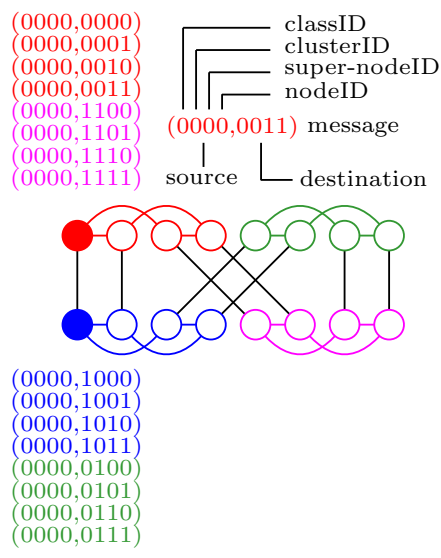

(a) Send message via cross-edge

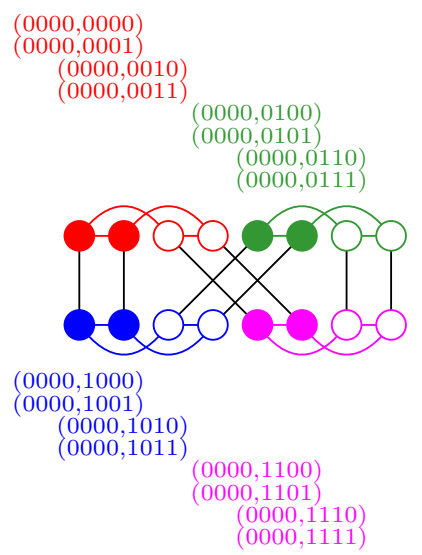

(c) Send message via cross-edge

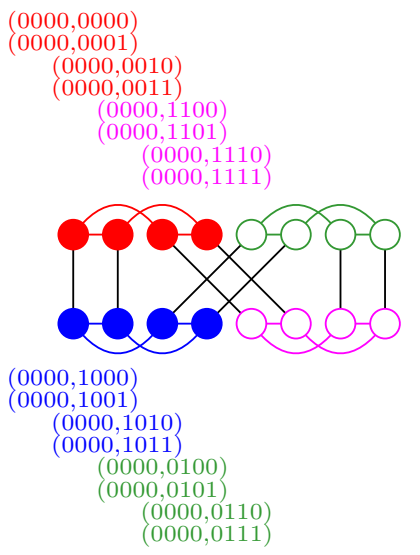

(b) Total exchange inside cluster

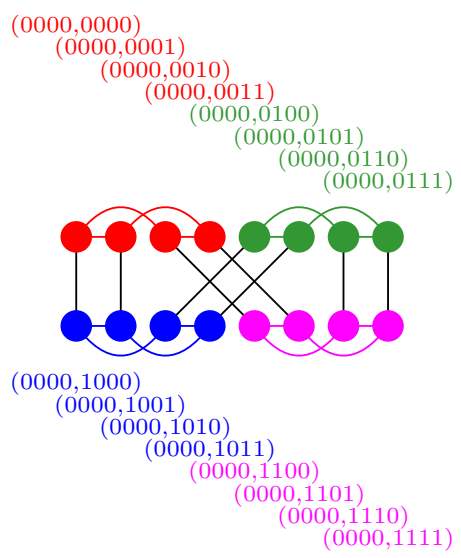

(d) Total exchange inside cluster

Fig. 2. Four stages of total exchange on $\operatorname{HDN}(B, 1, S)$ (only shows the case of node 0 ) 


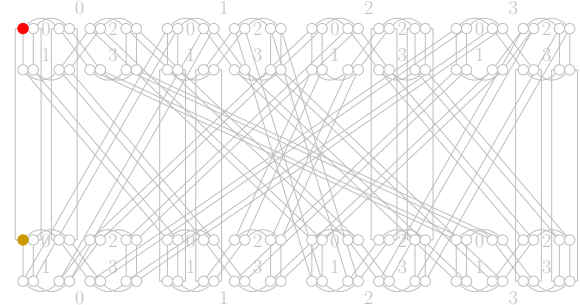

(a) Send message via cross-edge

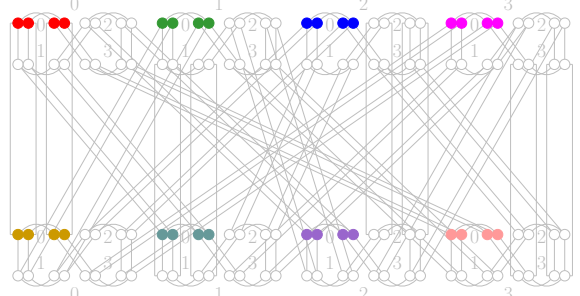

(c) Send message via cross-edge

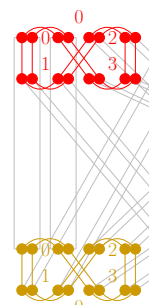

(b) Total exchange inside cluster

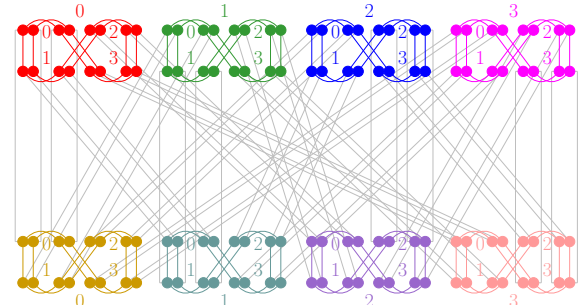

(d) Total exchange inside cluster

Fig. 3. Four stages of total exchange on $\operatorname{HDN}(B, 2, S)$ (only shows the case of node 0 )

described in four stages. Note that all nodes in the figures do the same operations but only the cases of node 0 are shown for clarity. Also note that the commas of node address in Figure 2 are omitted for saving spaces.

1. In the first stage, we first divide $M_{m y \_i d}$ into two parts, $M 1_{m y \_i d}$ and $M 2_{m y \_i d}$, where $M 1_{m y \_i d}$ contains all messages to be sent to the nodes in the clusters of my_type, and $M 2_{m y \_}$contains the rest message. Then, the first part of personalized messages $M 1$ is exchanged between my_id and partner, the neighbor via cross-edge of level $k$. The time this stage takes is $t_{s}+t_{w} m n_{k} / 2$.

2. In the second stage, we first pack all messages that are to be sent to the nodes in the cluster of level $k$ with clusterID $=q$ into a single message $m s g_{q}$. Then, we perform total exchange inside each cluster, where $m s g_{q}$ is to be sent to node with nodeID $=q$. The time this stage takes is denoted as $T_{k-1}\left(n_{k} / n_{k-1}\right)$, the time for total exchange inside the cluster.

3 . In the third stage, each node packs the received messages into a single message of length $n_{k} m$ and sends the packed message to its neighbor along the cross-edge of level $k$. After receive the message, each node unpacks the message received from its neighbor into $n_{k-1}$ messages, $m s g_{q^{\prime}}$, where $m s g_{q^{\prime}}$ is the collection of all messages destinated to node with nodeID $=q^{\prime}$ in the cluster. The time this stage takes is also $t_{s}+t_{w} m n_{k} / 2$. 
4. In the last stage, we perform total exchange again within each cluster of level $k$. This can be done since the packed messages sent through the level- $k$ cross-edge are all destinated to the nodes inside the cluster. The time this stage takes is also $T_{k-1}\left(n_{k} / n_{k-1}\right)$, which is the time for total exchange inside the cluster.

The algorithm is showed in Algorithm 2. All nodes execute the algorithm concurrently. In Algorithm 4, my_id is the $i d$ of the node. The initial message to be sent is $M_{m y_{-} i d}$ which contains $p$ messages of length $m$. At the end of the algorithm, each node stores the collection of all $p$ messages in result.

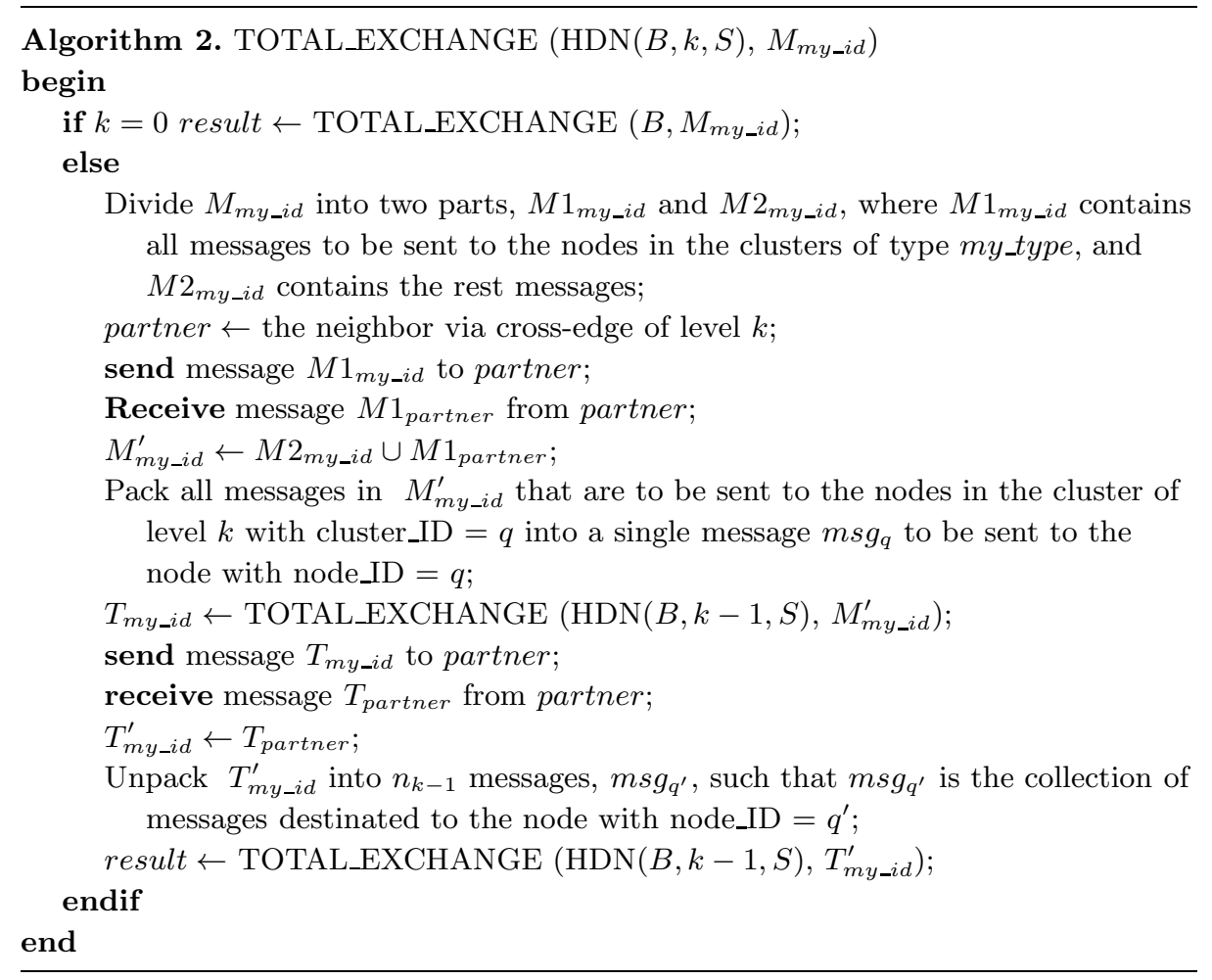

The time to complete the total exchange on an $\operatorname{HDN}(B, 1, S)$ is

$$
\begin{aligned}
T_{1}(m) & =\left(t_{s}+m t_{w} n_{1} / 2\right)+T_{0}\left(m n_{1} / 2\right)+\left(t_{s}+m t_{w} n_{1} / 2\right)+T_{0}\left(m n_{1} / 2\right) \\
& =2\left(t_{s}+m t_{w} n_{1} / 2\right)+2 T_{0}\left(m n_{1} / 2\right) .
\end{aligned}
$$

Generally, on an $\operatorname{HDN}(B, k, S)$, the time to complete the total exchange is

$$
\begin{aligned}
T_{k}(m) & =\left(t_{s}+t_{w} m n_{k} / 2\right)+T_{k-1}\left(n_{k} / n_{k-1}\right)+\left(t_{s}+t_{w} m n_{k} / 2\right)+T_{k-1}\left(n_{k} / n_{k-1}\right) \\
& =2\left(t_{s}+t_{w} m n_{k} / 2\right)+2 T_{k-1}\left(n_{k} / n_{k-1}\right) . \text { That is, } \\
T_{k}(m) & =\left(2^{k+1}-2\right)\left(t_{s}+t_{w} m n_{k} / 2\right)+2^{k} T_{0}\left(m n_{k} / 2\right)
\end{aligned}
$$


where $n_{k}$ is the total number of nodes and $T_{0}(m)$ is the time complexity for total exchange in $B$. In the examples of Figures 2 and 3 where $B$ is a 2-cube, $T_{0}(m)=2\left(t_{s}+t_{w} m\right)$. If $B$ is an $n$-cube, then $T_{0}(m)=n\left(t_{s}+t_{w} m 2^{n} / 2\right)$.

For the $\operatorname{HDN}(B, 1, S)$ shown in Figures 2, $T_{1}=2\left(t_{s}+8 t_{w} m\right)+4\left(t_{s}+8 t_{w} m\right)=$ $6\left(t_{s}+8 t_{w} m\right)$. In contrast, $T=4\left(t_{s}+8 t_{w} m\right)$ for a 4 -cube of same size. For the $\operatorname{HDN}(B, 2, S)$ shown in Figures 3 , $T_{2}=6\left(t_{s}+64 t_{w} m\right)+4 \times 2\left(t_{s}+64 t_{w} m\right)=$ $14\left(t_{s}+64 t_{w} m\right)$. In contrast, $T=7\left(t_{s}+64 t_{w} m\right)$ for a 7 -cube of same size. The times of total exchange for HDNs are longer than that for hypercubes but an HDN has much less links than a hypercube of the same size. We summarize this result in the following theorem.

Theorem 3. Assume that the time complexity $T_{0}(m)$ for total exchange in the base network $B$ is known, where $m$ is the length of each message. The time complexity $T_{k}(m)$ for total exchange on an $\operatorname{HDN}(B, k, S), k>0$, is $T_{k}(m)=$ $\left(2^{k+1}-2\right)\left(t_{s}+t_{w} m n_{k} / 2\right)+2^{k} T_{0}\left(m n_{k} / 2\right)$, where $n_{k}=\left(2 n_{0}\right)^{2^{k}} /\left(2 \prod_{i=1}^{k} s_{i}\right)$.

\section{Concluding Remarks}

The hierarchical dual-net can connect a large number of nodes with a small nodedegree and a short diameter. It is a potential candidate for the interconnection network of the supercomputers of the next generation that may have more than one million of nodes. We can select a popular network of small size that is a product graph as the base network and then connect multiple base modules with cross links (cables) to construct a very large-scale hierarchical dual-net. We can also select a suitable set of integers based on the base network to control the number of nodes in the supercomputer. The base networks can be implemented in a NoC VLSI and high-speed line cables may be used as the cross links to connect PCB modules in cabinets. We presented an efficient algorithm for total exchange on recursive dual-net. There are many problems, such as disjoint path and fault-tolerant routing, on recursive dual-net that are worth further research.

\section{References}

1. Adiga, N.R., Blumrich, M.A., Chen, D., Coteus, P., Gara, A., Giampapa, M.E., Heidelberger, P., Singh, S., Steinmacher-Burow, B.D., Takken, T., Tsao, M., Vranas, P.: Blue gene/l torus interconnection network. IBM Journal of Research and Development 49(2/3), 265-276 (2005)

2. Beckman, P.: Looking toward exascale computing, keynote speaker. In: International Conference on Parallel and Distributed Computing, Applications and Technologies (PDCAT 2008), Dunedin, New Zealand (December 2008)

3. Ghose, K., Desai, K.R.: Hierarchical cubic networks. IEEE Transactions on Parallel and Distributed Systems 6(4), 427-435 (1995)

4. IBM: Roadrunner: Hardware and Software Overview. IBM Corporation (January 2009), http://www.redbooks.ibm.com/redpapers/pdfs/redp4477.pdf

5. Kumar, V., Grama, A., Gupta, A., Karypis, G.: Introduction to parallel computing: design and analysis of algorithms. Benjamin/Cummings Press (1994) 
6. Li, Y., Peng, S., Chu, W.: Efficient collective communications in dual-cube. The Journal of Supercomputing 28(1), 71-90 (2004)

7. Li, Y., Peng, S., Chu, W.: Recursive dual-net: A new universal network for supercomputers of the next generation. In: Hua, A., Chang, S.-L. (eds.) ICA3PP 2009. LNCS, vol. 5574, pp. 809-820. Springer, Heidelberg (2009)

8. Li, Y., Peng, S., Chu, W.: Hierarchical dual-net: A flexible interconnection network and its routing algorithm. In: Proceedings of the Second International Conference on Networking and Computing, Osaka, Japan, pp. 58-67 (November 2011)

9. Preparata, F.P., Vuillemin, J.: The cube-connected cycles: a versatile network for parallel computation. Commun. ACM 24, 300-309 (1981)

10. TOP500: Supercomputer Sites (June 2013), http://top500.org/ 